Zuh S.G., Nagy Ö., Zazgyva Ancuța, Russu O.M., Gergely I., Pop T.S.

\title{
Correlations between the Harris Hip Score and the Visual Analogue Scale in the assessment of total hip replacement in hip dysulasia
}

Department of Orthopaedics and Traumatology, University of Medicine and Pharmacy

Tîrgu Mureș, Romania

\begin{abstract}
Total hip replacement is one of the most frequently performed orthopaedic interventions that can significantly improve the functional status and the quality of life of patients suffering from hip arthrosis. Recently patient satisfaction and patient-reported results of total hip arthroplasty are increasingly emphasised as important tools for the assessments of these interventions. For patients with arthrosis secondary to hip dysplasia, these evaluations can be more difficult, due to younger age and higher functional demands. In this study we compared the Visual Analogue Scale and the Harris Hip Score in order to determine the correlations between these instruments and analyse the possibility of replacing the Harris Score with the Visual Analogue Scale in evaluating the results of hip surgery in patients with dysplastic hips. Our study included 37 women and 4 men (53 hips), with a mean age of 50.96 years (35-58 years), followed for a mean of 4 years postoperatively. Both assessment instruments were used preoperatively and at the follow-up visits. We observed a positive correlation of the values of the Visual Analogue Scale and the Harris Hip Score both preoperatively and
\end{abstract}

\section{Gergely I.}

Mihai Viteazul 31

Tîrgu Mureș

gergelyistvan@studium.ro

0745360293 postoperatively, with correlation coefficients of +0.71 $(\mathrm{P}<0.001)$ and $+0.77(\mathrm{P}<0.001)$ respectively. Given these positive correlations, we assumed that the Visual Analogue Scale could replace the Harris Hip Score in the evaluation of patients after total hip replacement. Still it is recommended to combine the Visual Analogue Scale with objective measurements and radiological examination in order to identify significant postoperative changes.

Keywords: hip dysplasia, total hip replacement, Visual Analogue Scale, Harris Hip Score

\section{Introduction}

Total hip replacement (THR) can significantly improve both the functional status and the quality of life of patients suffering from hip arthrosis, especially in the more advanced stages. Clinical decisions regarding THR, as well as interpreting the results of the intervention and assessing the costs and indications can vary in different settings[1].

The World Health Organization (WHO) defined quality of life as a multidimensional model that depends on the person's physical, psychological, social and emotional state and involves the capacity to undertake activities of daily living[2]. As such, quality 
of life - and its changes after different treatments - is difficult to assess. In the case of THR for hip dysplasia, the evaluation of changes in the quality of life of treated patients is more difficult compared to that of patients with primary hip arthrosis, due to the special functional and clinical state of the first (related to the younger age at surgery).

A decade ago the results of THR were usually assessed by studying morbidity, mortality, complications and wear rates, but currently implant success or failure is not the only aspect to consider. There is an increasing emphasis on patient satisfaction and improvement of the quality of life. Thus, specific articular measurements need to be correlated with the patient's general state of health and comorbidities[3]. As the main purpose of THR is to improve the quality of life of patients, it becomes obvious that a better understanding of the effects of the intervention requires an evaluation of these factors[4].

Although pain is a complex symptom that is difficult to evaluate, the Visual Analogue Scale (VAS) is a simple assessment tool that can be used to assess the intensity of pain[5]. In a similar manner, patient satisfaction after THR is also a complex factor, but assessing it can help in proving treatment efficacy[6, 7]. The patient is the most important person affected by the intervention, so modern healthcare systems include patient opinion in the assessment of the results of THR[8-10].

Developed for evaluating hip surgery results, the original version of the Harris Hip Score (HHS) was published in 1969[11]. It can be used for planning treatment and determining the optimal timing of the intervention, and also for clinical follow-up with the assessment of functional and symptomatic improvement.

In our study we compared these two popular tools used for assessing the results of THR - VAS and HHS - in a series of patients suffering from developmental dysplasia of the hip (DDH) and that have undergone THR. We aimed to determine the correlations between these instruments and analyse the possibility of replacing HHS with the VAS in evaluating the results of hip surgery in these patients.

\section{Materials and methods}

A number of 485 uncemented total hip arthroplasties were performed in the Clinic of Orthopaedics and Traumatology of Târgu Mureş between January 2007 and January 2011. In our study we only included patients treated for hip arthrosis secondary to DDH, so the final assessment was made on a group of 41 patients (53 hips), with a mean age of 50.96 years (35-58 years), 37 women and 4 men.

Patients were followed for a mean of 4 years postoperatively, with follow-up visits at 3, 6 and 12 months after surgery and yearly thereafter. Assessment was based on the VAS and HHS[11], used preoperatively and at the postoperative visits. We also noted the evolution of symptoms, the radiological aspect of the hip and the need for revision surgery. Clinical success was defined as the reduction or disappearance of pain in the hip.

The evaluation of hip surgery results is based on three essential clinical parameters: pain, hip joint mobility (especially flexion), and hip function. The patient's own assessment of the results can be a very important factor to consider, and the best way to obtain it is by using the VAS. Thus the patient will indicate his/ her perception of the evaluated item, in the absence of the examiner. Pain or the degree of satisfaction can be evaluated this way, choosing between the values of 0 (maximum intensity of pain or totally dissatisfied) and 10 (no pain or very pleased). Other items can also be assessed with the VAS, and each segment can further be divided into 10 parts, so that the final value will vary between 0 and 100 .

The second measurement instrument used by us was the HHS. This is one of the most commonly used clinical assessment tools. Unfortunately it does not correlate the patient's pain with activity, describing pain only as absent, mild, moderate, etc. We recorded the total value of the HHS at all follow-up visits. Our goal was to find correlations between HHS and the VAS. For statistical analysis of the collected data we used Analyse-it ${ }^{\circledR}$ software, Standard Edition, version 3.0. 


\section{Results}

We followed patients for a minimum of 2 years postoperatively, with a mean follow-up of 4 years ( 2 to 6 years). Figure 1 shows patient distribution based on the severity of hip dysplasia according to the Crowe classification system: 29 Crowe I hips, 20 Crowe II hips and 4 of type Crowe III.

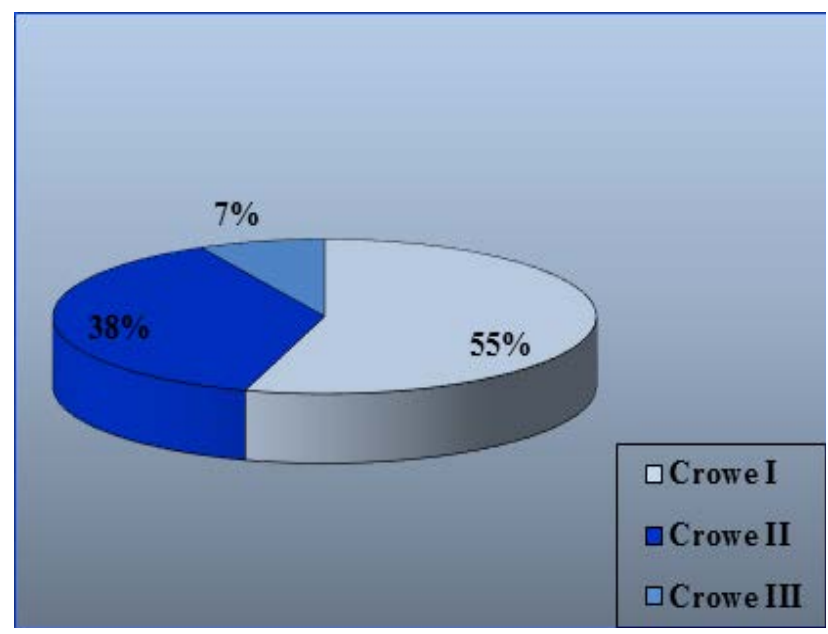

Figure 1. - Distribution of patients according to the severity of hip dysplasia (Crowe classification)

The mean value of the HHS increased from 45.94 points (SD 5.19) before surgery (range 38-55 points) to 93.81 points (SD 7.12) - (ranging from 61 to 100 points ) at the last evaluation. Similarly VAS values increased from a mean of 42.35 (SD 8.67) preoperatively to 93.67 (SD 8.77) postoperatively. Calculations were performed using the mentioned statistical software, and the values are presented in tables I and II. Pain intensity showed a significant decrease after surgery, as measured with the VAS.

Table I-Descriptive statistics of the preoperative values of the Harris Hip Score and Visual Analogue Scale

\begin{tabular}{|lccccc|}
\multicolumn{1}{|c}{ Variable } & Mean & SD & Minim & Median & Maxim \\
\hline $\begin{array}{l}\text { Preoperative } \\
\text { HHS }\end{array}$ & 45.94 & 5.19 & 38 & 45.0 & 55 \\
\hline $\begin{array}{l}\text { Preoperative } \\
\text { VAS }\end{array}$ & 42.35 & 8.67 & 20 & 45.0 & 55 \\
\hline
\end{tabular}

Of the 41 patients, 4 still had a slight limp at the last follow-up, but it was improved compared to the preoperative limp. In 50 cases we found pain to have completely disappeared - for the remainder of 3 hips there were complaints of pain of a variable intensity, but these improved significantly when patients started using walking aids (cane, crutches), so reintervention was not deemed necessary. One patient had pain in the hip and thigh - her symptomatology was associated with a significant limitation of daily activities and radiological signs of mobilization of the femoral component. In this case it is worth noting pain location and intensity, with radiation to the thigh and knee - this patient needed re-intervention, with revision of the femoral component.

Table II - Descriptive statistics of the postoperative values of the Harris Hip Score and Visual Analogue Scale

\begin{tabular}{lccccc}
\multicolumn{1}{c}{ Variable } & Mean & SD & Minim & Median & Maxim \\
$\begin{array}{l}\text { Postoperative } \\
\text { HHS }\end{array}$ & 93.81 & 7.12 & 61 & 95.0 & 100 \\
\hline $\begin{array}{l}\text { Postoperative } \\
\text { VAS }\end{array}$ & 93.67 & 8.77 & 40 & 95.0 & 100 \\
\hline
\end{tabular}

Regarding the statistical analysis of data, the type of relationship between the obtained data determines the methods of measurement and type of appropriate association tests. In case of a linear relationship between different variables, their correlation is represented by a bivariate normal density ellipse. When the studied variables are highly correlated, the ellipse is narrow; if the variables are uncorrelated, the ellipse is wider and more rounded. A linear relationship between the studied variables involves the use of the product-moment correlation coefficient (or Pearson coefficient) to describe the strength of the linear relationship. The value of this coefficient can vary from -1 (indicating a perfect negative correlation) to +1 (perfect positive correlation indicator), with 0 meaning that the variables are not correlated. 


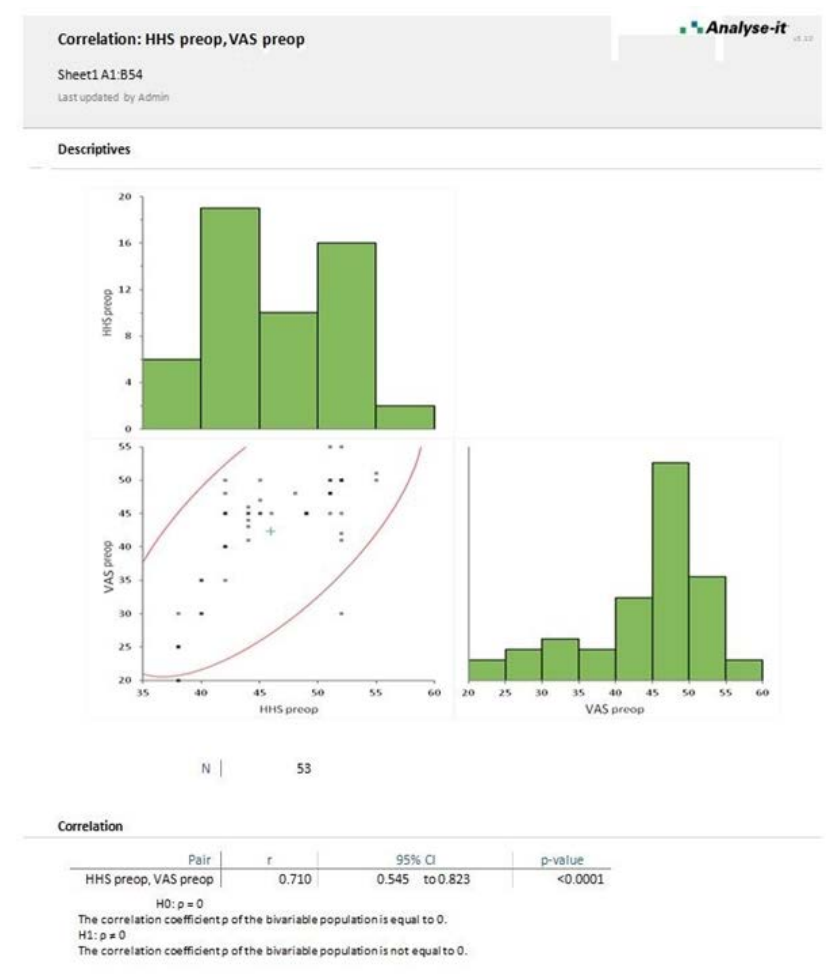

Figure 2. - Statistical analysis of the correlation between the preoperative values of the Harris Hip Score and the Visual Analogue Scale

Correlating the preoperative values of HHS and VAS, we obtained a Pearson coefficient of 0.71 $(\mathrm{P}<0.001)$ - the results are shown in figure 2 . We thus obtained a positive correlation between HHS and VAS preoperatively.

When correlating the averages of HHS and VAS values obtained postoperatively, the correlation coefficient was $0.77(\mathrm{P}<0.001)$, meaning a positive correlation between the values of HHS and VAS postoperatively, as shown in figure 3 .

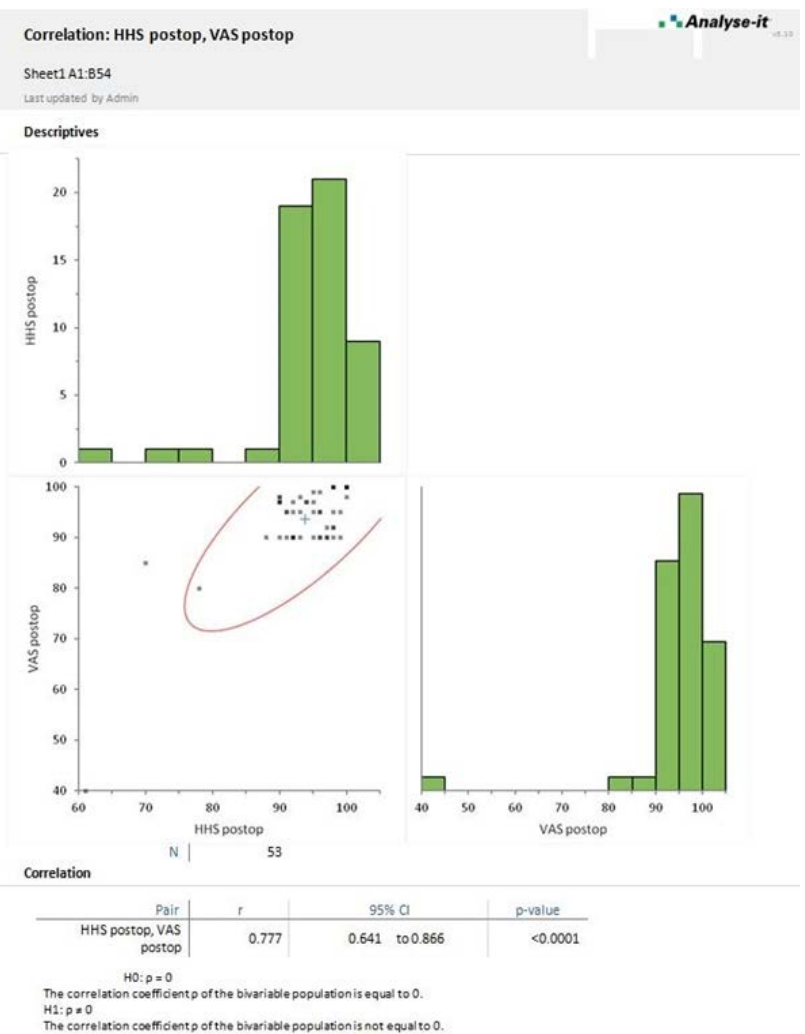

Figure 3. - Statistical analysis of the correlation between the postoperative values of the Harris Hip Score and the Visual Analogue Scale

\section{Discussion}

The results of surgical interventions are visible and practical effects. Measuring and evaluating these visible effects is particularly important for studying and defining standards for medical practice. Assessment tools should be quick and easy to use, reproducible, specific to the question that is being evaluated, applicable and cost-effective.

In 1931 Fergusson and Howarth[12] developed the first assessment tools for evaluating articular mobility of the hip joint. For patients suffering from femoral epiphysiolysis, more points were given for flexion and abduction as compared to adduction and 
hyperextension. This instrument later served as a base for Merle d'Aubigne and Postel's scoring system published in 1954[13] and that was subsequently modified by Charnley in 1972[14].

The Harris Hip Score[11] was created in 1969 in order to evaluate pain, function, range of motion and the presence/ absence of hip joint deformities. In 1972, Andersson[15] pointed out the problems related to the variation and reproducibility of the available evaluation tools, and demonstrated that the 9 measurement systems used for the same patient population could be divided into 5 statistically significant groups. Currently, the most used evaluation instruments for the hip joint are the HHS and the Merle d'Aubigné and Postel score as modified by Charnley. Both of these combine patientspecific assessment elements with those graded by the surgeon. The Charnley score includes pain, joint mobility and gait, and uses a scale ranging from 0 to 6 , but these elements are not combined into a total score. In contrast, the HHS - which is currently one of the most commonly used methods for evaluating the results of total hip replacement - combines sub-scores for pain (0-44 points), functionality ( 0 to 47 points, evaluated based on the presence/ absence of a limp, use of auxiliary walking aids for support, and specific activities), joint range of motion ( 0 to 5 points), and the presence/ absence of hip joint deformities ( 0 to 4 points) into one final value.

It has been demonstrated that HHS has a higher sensitivity in evaluating the results of THR as compared to generic rating scales such as the SF-36 (Short Form Health Survey)[11, 16-21], although the latter showed good sensitivity in studies on the effectiveness of different medical interventions in osteoarthritis, such as medical gymnastics, pharmacological treatment and total hip arthroplasty[17, 19, 22, 23].

Hoeksma et al.[24] compared the sensitivity of clinical assessment tools for hip function in patients with osteoarthritis of the hip (HHS, SF-36 subscales, walking speed and walking pain tests), concluding that the HHS is useful for evaluating treatment, including recovery interventions such as medical gymnastics and manual therapy, especially given that both recovery interventions studied focus on the factors evaluated by the HHS - pain, range of motion and function. From all the evaluation instruments included in the study, HHS was the best in describing hip function, and it was also the only one to combine elements graded by the surgeon with those selfreported by the patient - this could explain the higher sensitivity of HHS.

Ideally, the assessment tools for THR results must have a proven validity and reproducibility; they also must be cheap, easy and quick to use, as well as easily understood by the doctor and patient. It is highly desirable to obtain score values directly, without the need for complicated calculations. Patient compliance is higher when using short, simple questionnaires that assess the patient's degree of satisfaction after specific interventions; therefore simple questions on satisfaction are an ideal compromise between detailed and complicated measuring instruments of the state of health on one hand and the need to generate high response rates to avoid errors of assessment on the other hand. The simple questionnaires are also suitable for monitoring standards of practice.

The VAS is a simple instrument for assessing variations in pain intensity [5], and it is frequently used in practice, and reported in the literature. Assessment of pain and the degree of satisfaction after THR is difficult due to their complex nature that is influenced by emotional, cognitive, physical, sensory, subjective, behavioural and socio-cultural elements.

In 1987 Guyatt et al.[25] compared the VAS with the Likert scale (a nominal scale used in psychometrics) and showed a greater variability in the degrees of improvement of the results when using VAS, concluding that VAS is more appropriate for assessing patient satisfaction. In addition, Haverkamp et al.[26] showed that the use of a single question from the Likert scale for evaluating satisfaction is invalid, which lead to the introduction and use of VAS for satisfaction assessment[27].

In the absence of simple, validated and practical patient satisfaction scoring systems to be used after arthroplasty in the clinical setting, Brokelman et al.[28] studied and demonstrated the validity and reproducibility of VAS as an assessment tool to quantify patient satisfaction after THR. The authors recommend using satisfaction VAS only in association with other objective and subjective evaluation instruments.

The Visual Analogue Scale presents a number 
of advantages over the HHS in evaluating bilateral hip arthroplasty, as well as in the case of generalized joint diseases. First of all, it is much simpler to use, and secondly, the patient can focus his/ her attention on the hip that is being evaluated, as opposed to the HHS that may be influenced by the condition of the contralateral hip and by any comorbidities[29].

The VAS is a purely subjective assessment tool that needs to be complemented by objective evaluation. One of the most appropriate and simple objective assessments that can be combined with VAS is the measurement of the degrees of hip flexion[29]. In addition, follow-up radiological examinations are important for early detection of asymptomatic cases of osteolysis or endoprosthetic component migration. other objective and subjective evaluation tools.

By comparing two of the most frequently used tools for evaluating the results of cementless hip arthroplasty in patients treated for hip arthrosis secondary to $\mathrm{DDH}$, we observed a positive correlation of the results obtained with these instruments both preoperatively and postoperatively, with correlation coefficients of $+0.71(\mathrm{P}<0.001)$ and $+0.77(\mathrm{P}<0.001)$. Given the positive correlation between VAS and HHS, it can be assumed that VAS could replace HHS in the evaluation of patients after THR, but it is desirable to use this assessment tool in association with objective measurements and radiological examination that can identify postoperative changes of the implant and osseous structures.

\section{Conclusions}

Assessing changes in quality of life after THR for the treatment of hip arthrosis secondary to DDH is hampered by the fact that these patients have special clinical and functional characteristics that are different from those of patients treated for primary hip arthrosis, partly due to the usually younger age at which DDH leads to THR. The Harris Hip Score is a useful tool not only for the monitoring of patients and evaluation of clinical and functional improvements after treatment, but also for planning management and determining the optimal timing of surgery.

The Visual Analogue Scale is a simple tool for assessing changes in pain intensity and it is commonly used both in clinical practice and in various studies. VAS is easily understood by the patient and it focuses on the element evaluated, without being influence by comorbidities or the status of the contralateral hip - therefore it seems more appropriate for assessing patient satisfaction after THR. Although the validity and reproducibility of satisfaction VAS as a tool to quantify the degree of patient satisfaction after THR have already been demonstrated, it is still recommended to be used only in combination with

\section{References}

1. Quintana J.M., Arostegui I., Azkarate J., Goenaga J.I., Elexpe X., Letona J. \& Arcelay A. (2000). Evaluation of explicit criteria for total hip joint replacement. Journal of clinical epidemiology. 53(12), 1200-1208.

2. The World Health Organization Quality of Life Assessment (WHOQOL). (1998). Development and general psychometric properties. Social science \& medicine. 46(12), 1569-1585.

3. Ahmad M.A., Xypnitos F.N. \& Giannoudis P.V. (2011). Measuring hip outcomes: common scales and checklists. Injury. 42(3), 259-264.

4. Xu M., Garbuz D.S., Kuramoto L. \& Sobolev B. (2005). Classifying health-related quality of life outcomes of total hip arthroplasty. $B M C$ musculoskeletal disorders. 6, 48.

5. Chapman C.R., Casey K.L., Dubner R., Foley K.M., Gracely R.H. \& Reading A.E. (1985). Pain measurement: an overview. Pain. 22(1), 1-31.

6. Knahr K., Kryspin-Exner I., Jagsch R., Freilinger W., Kasparek M. (1998). Evaluating the quality of life before and after implantation of a total hip endoprosthesis. Zeitschrift fur Orthopadie und ihre Grenzgebiete. 136(4), 321-329. 
7. Lingard E., Hashimoto H. \& Sledge C. (2000). Development of outcome research for total joint arthroplasty. Journal of orthopaedic science: official journal of the Japanese Orthopaedic Association. 5(2), 175-177.

8. Amadio P.C. (1993). Outcomes measurements. The Journal of bone and joint surgery American volume. 75(11), 1583-1584.

9. Lieberman J.R., Dorey F., Shekelle P., Schumacher L., Thomas B.J., Kilgus D.J. \& Finerman G.A. (1996). Differences between patients' and physicians' evaluations of outcome after total hip arthroplasty. The Journal of bone and joint surgery American volume. 78(6), 835838.

10. Wright J.G. \& Young N.L. (1997). The patientspecific index: asking patients what they want. The Journal of bone and joint surgery American volume. 79(7), 974-983.

11. Harris W.H. (1969). Traumatic arthritis of the hip after dislocation and acetabular fractures: treatment by mold arthroplasty. An end-result study using a new method of result evaluation. The Journal of bone and joint surgery American volume. 51(4), 737-755.

12. Fergusson A. \& Howarth M. (1931). Slipping of the upper femoral epiphysis. JAMA. (97), 18671872.

13. D'Aubigne R.M. \& Postel M. (1954). Functional results of hip arthroplasty with acrylic prosthesis. The Journal of bone and joint surgery American volume. 36-A(3), 451-475.

14. Charnley J. (1972). The long-term results of low-friction arthroplasty of the hip performed as a primary intervention. The Journal of bone and joint surgery British volume. 54(1), 61-76.

15. Andersson G. (1972). Hip assessment: a comparison of nine different methods. The Journal of bone and joint surgery British volume. 54(4), 621-625.

16. Shields R.K., Enloe L.J., Evans R.E., Smith K.B. \& Steckel S.D. (1995). Reliability, validity, and responsiveness of functional tests in patients with total joint replacement. Physical therapy. 75(3), 169-176; discussion 176-169.

17. Kosinski M., Keller S.D., Ware J.E., Jr., Hatoum H.T. \& Kong S.X. (1999). The SF-36
Health Survey as a generic outcome measure in clinical trials of patients with osteoarthritis and rheumatoid arthritis: relative validity of scales in relation to clinical measures of arthritis severity. Medical care. 37(5 Suppl), MS23-39.

18. Wright J.G. \& Young N.L. (1997). A comparison of different indices of responsiveness. Journal of clinical epidemiology. 50(3), 239-246.

19. Angst F., Aeschlimann A., Steiner W. \& Stucki G. (2001). Responsiveness of the WOMAC osteoarthritis index as compared with the SF36 in patients with osteoarthritis of the legs undergoing a comprehensive rehabilitation intervention. Annals of the rheumatic diseases. 60(9), 834-840.

20. Bachmeier C.J., March L.M., Cross M.J., Lapsley H.M., Tribe K.L., Courtenay B.G. \& Brooks P.M. (2001). Arthritis Cost and Outcome Project Group. A comparison of outcomes in osteoarthritis patients undergoing total hip and knee replacement surgery. Osteoarthritis and cartilage/OARS, Osteoarthritis Research Society. 9(2), 137-146.

21. Anderson J.J., Firschein H.E. \& Meenan R.F. (1989). Sensitivity of a health status measure to short-term clinical changes in arthritis. Arthritis and rheumatism. 32(7), 844-850.

22. Davies G.M., Watson D.J. \& Bellamy N. (1999). Comparison of the responsiveness and relative effect size of the western Ontario and McMaster Universities Osteoarthritis Index and the short-form Medical Outcomes Study Survey in a randomized, clinical trial of osteoarthritis patients. Arthritis care and research: the official journal of the Arthritis Health Professions Association. 12(3), 172-179.

23. Norman G.R., Stratford P. \& Regehr G. (1997). Methodological problems in the retrospective computation of responsiveness to change: the lesson of Cronbach. Journal of clinical epidemiology. 50(8), 869-879.

24. Hoeksma H.L., Van Den Ende C.H., Ronday H.K., Heering A. \& Breedveld F.C. (2003). Comparison of the responsiveness of the Harris Hip Score with generic measures for hip function in osteoarthritis of the hip. Annals of the rheumatic diseases. 62(10), 935-938. 
25. Guyatt G.H., Townsend M., Berman L.B. \& Keller J.L. (1987). A comparison of Likert and visual analogue scales for measuring change in function. Journal of chronic diseases. 40(12), 1129-1133.

26. Haverkamp D., Sierevelt I.N., van den Bekerom M.P., Poolman R.W., van Dijk C.N. \& Marti R.K. (2008). The validity of patient satisfaction as single question in outcome measurement of total hip arthroplasty. Journal of long-term effects of medical implants. 18(2), 145-150.
27. Brokelman R.B., van Loon C.J. \& Rijnberg W.J. (2003). Patient versus surgeon satisfaction after total hip arthroplasty. The Journal of bone and joint surgery British volume. 85(4), 495-498.

28. Brokelman R.B., Haverkamp D., van Loon C., Hol A., van Kampen A. \& Veth R. (2012). The validation of the visual analogue scale for patient satisfaction after total hip arthroplasty. European orthopaedics and traumatology. 3(2), 101-105.

29. de Nies F. \& Fidler M.W. (1997). Visual analog scale for the assessment of total hip arthroplasty. The Journal of arthroplasty. 12(4), 416-419. 